\title{
Vom Steuern und Gegensteuern in der Sozialen Arbeit
}

\section{Bei einer offenen Dialogkultur können Systeme sich selbst steuern}

HARALD TORNOW

Dr. phil. Harald Tornow ist Diplom-

Psychologe, Master of TOM (Uni

Kaiserslautern), Systemischer

Familientherapeut und Psycho-

dramatherapeut. Er ist Leiter des

e/l/s-Instituts für Qualitätsent-

wicklung sozialer Dienstleistungen

in Wülfrath. Davor sammelte er

30 Jahre praktische Erfahrung in

der Jugendhilfe. Er ist Mitglied im

Vorstand des Evangelischen Erzie-

hungsverbands e. V. (EREV).

harald.tornow@els-institut.de

\author{
Komplexe Systeme zu planen, zu steuern und \\ zu prüfen ist eine Daueraufgabe in der Sozialen \\ Arbeit. Die Teile des Systems sind dabei genauso \\ bedeutsam wie das Umfeld, wie das Beispiel \\ der Hilfen zur Erziehung zeigen kann.
}

Dass jemand etwas steuert, ist eine alte Vorstellung. Seit Menschen die Tiere domestiziert und Maschinen ersonnen haben, gibt es das Problem, Bewegungen in eine gewollte Richtung zu lenken. Wagenlenker und Steuermänner auf Schiffen, Reiter und Hirten beherrschten und beherrschen die Kunst, eine willenlose Maschine oder eigenwillige Lebewesen in Bewegung zu setzen und sie dort ankommen an lassen, wohin sie ein Befehl, ein Plan oder allgemein gesagt ein Wille haben will.

Mit der Sesshaft-Werdung der Menschen und später der Bildung von Stadtstaaten wird die Steuerung von Menschen ein Problem. Auch vorher musste die Bewegung nomadisierender Menschengruppen gelenkt werden, aber wie viel größer war die Anforderung, wenn es jetzt galt, große Bauwerke zu errichten, Armeen aufzustellen und zu verpflegen, die Produktion von Gütern und den Warenverkehr zu steuern.

Denn Ordnung ist schon immer bedroht. Der geordneten, durchdachten Stadt von Gilgamesch steht der wilde Wald gegenüber (Sedlacek, 2012). Auch das Meer galt von Menschheitsgedenken an als Ort des Chaos, der Gefahr, des nicht Lenkbaren. Wellen und Stürme, die Wolken am Himmel, die Strudel in einem Flusslauf, die hinausgeschleuderten Sonnenwinde, die Kinetik der RNS-Moleküle in unseren Zellen, alles das folgt keinen Gesetzen, die eine Steuerung erlaubt.

Den Begriff Steuerung habe ich in diesem kurzen Exkurs für die Hand- lungen verwendet, mit denen bestimmte Elemente auf ein Ziel hinbewegt werden. Elemente können unbelebte Dinge, Maschinen oder Lebewesen sind. Die erzeugte Bewegung kann ein passives Bewegt-Werden sein (bei Dingen), sie können technische Vorgänge innerhalb von Maschinen sein oder sie können Handlungen von Tieren oder Menschen sein.

Zwischen dem Chaos und der einfachen Mechanik liegen komplexe Systeme, deren Elemente nicht völlig zufällig und ungeordnet, aber auch nicht kausal determiniert sind. Diese Grenzstrukturen sind es, die Lenker, Steuerer, Willens- und Machtmenschen so herausfordern, weil sie sehr wohl reagieren, aber nie genau so, wie man beabsichtigt.

\section{Von der Steuerfähigkeit lebendiger Systeme}

Komplexe lebendige Systeme haben noch eine weitere, irritierende und aus Sicht derer, die steuern wollen, frustrierende Eigenschaft: Sie steuern sich selbst aus sich heraus und verändern sich ohne äußeren Anlass. Alle biologischen und sozialen Systeme sind lebendig und komplex. Sind sie überhaupt steuerbar?

Im Sinne einer technisch-rationalen Steuerung sind sie das sicherlich nicht, allenfalls sind sie mehr oder weniger erfolgreich beeinflussbar. Komplexe lebendige Systeme reagieren auf äußere Einflüsse. Es kann aber auch sein, dass sich trotz aller Versuche, Bewegungen 
oder Änderungen hervorzurufen gar nichts tut.

Solche Systeme erhalten den derzeitigen Zustand homöostatisch oder sie kehren nach einem Zustand der Irritation oder kurzfristiger Anpassung wieder in den alten Zustand zurück. Auch dieses sind Erfahrungen, die Planern, Entwicklern, Modernisierern und anderen Promotoren gar nicht recht sind. Sie nennen das Widerstand.

Welche Strategien verfolgen die neuen Steuerer in der Sozialen Arbeit, hier am Beispiel von Hilfen zur Erziehung? In einem Aufsatz des Nachrichtendienstes des Deutschen Vereins (Tornow, 2005) habe ich vier Ebenen unterschieden, seinerzeit insbesondere mit dem Hinweise, niemand solle anderen auf der nächsten Ebene ins Steuerrad greifen (vgl. Tabelle).

Die Ebenen sind hierarchisch angeordnet in dem Sinne, dass die obere Ebene immer die unteren umfasst. Eine Macht-Hierarchie kann daraus nicht abgeleitet werden. In modernen Gesellschaften und Organisationen ist es eher umgekehrt, zumindest dem theoretischen Anspruch nach, dass die Klienten definieren, worin für sie eine Dienstleistung besteht. Moderne Organisationen verstehen sich nicht als kleine Herrschaften über ihre Mitarbeitende, sondern als Dienstleister für die Mitarbeiterinnen und Mitarbeiter um diese mit dem zu versorgen, was sie brauchen, um einen guten Job für die Klienten zu machen: "form follows function, structure follows process «. Genauso sollte das politisch-administrative System die Rahmenbedingungen setzen, unter denen es Institutionen gut schaffen, Professionellen solche Arbeitsbedingungen zu bieten, die geeignet sind, Wirkungen für Adressaten und für die Gesellschaft zu erzeugen.

\section{Warum die Politik steuern will}

Wenn ich das richtig sehe, ist der Beweggrund für die Steuerungsbemühungen der oberen Ebene in der Jugendhilfe auf der einen Seite ein Maximum an Chancengerechtigkeit, Funktionsfähigkeit in Familien, Kindeswohl, Integration und Bildung zu erreichen, auf der an-

\begin{tabular}{|c|c|c|c|}
\hline \multicolumn{2}{|c|}{$\begin{array}{l}\text { Steuerungsebenen für personen- } \\
\text { bezogene soziale Dienstleistungen }\end{array}$} & \multirow[b]{2}{*}{$\begin{array}{l}\text { Leitungskräfte des } \\
\text { Leistungsträgers, } \\
\text { Leistungsanbieter }\end{array}$} & \multirow[b]{2}{*}{$\begin{array}{l}\text { Professionelle } \\
\text { Helfer }\end{array}$} \\
\hline $\begin{array}{l}\text { Akteure } \\
\text { System-Ebene }\end{array}$ & $\begin{array}{l}\text { Gesellschaftliche / } \\
\text { Politische Macht- } \\
\text { träger }\end{array}$ & & \\
\hline $\begin{array}{l}\text { Politisch- } \\
\text { administrativ: } \\
\text { Staat, Gesellschaft }\end{array}$ & $\begin{array}{l}\text { Aufgabe: sozial- } \\
\text { staatliche Ziele } \\
\text { definieren, Rahmen } \\
\text { setzen, Ressourcen } \\
\text { verteilen }\end{array}$ & $\begin{array}{l}\text { Interesse: für die } \\
\text { eigene Tätigkeit } \\
\text { förderliche Rah- } \\
\text { menbedingungen }\end{array}$ & $\begin{array}{l}\text { Interesse: förder- } \\
\text { liche Rahmenbedin- } \\
\text { gungen }\end{array}$ \\
\hline $\begin{array}{l}\text { Manageriell: } \\
\text { Unternehmen, } \\
\text { Organisation }\end{array}$ & $\begin{array}{l}\text { Interesse: Zieler- } \\
\text { reichung; Einhalten } \\
\text { von Gesetzen, } \\
\text { Verordnung, Ver- } \\
\text { einbarung }\end{array}$ & $\begin{array}{l}\text { Aufgabe: Struktur } \\
\text { und Prozessorgani- } \\
\text { sation so gestalten, } \\
\text { dass Effektivität } \\
\text { und Effizienz opti- } \\
\text { miert sind }\end{array}$ & $\begin{array}{l}\text { Interesse: förder- } \\
\text { liche Rahmenbedin- } \\
\text { gungen }\end{array}$ \\
\hline $\begin{array}{l}\text { Professionell: } \\
\text { Helfende } \\
\text { Interaktion }\end{array}$ & $\begin{array}{l}\text { Interesse: Effektivi- } \\
\text { tät der Fachlichkeit, } \\
\text { Transparenz des } \\
\text { Vorgehens, Zuver- } \\
\text { lässigkeit - keine } \\
\text { Fehler }\end{array}$ & $\begin{array}{l}\text { Interessen: Umset- } \\
\text { zung der Leistungs- } \\
\text { zusagen, Umsetzen } \\
\text { der Unternehmens- } \\
\text { strategie }\end{array}$ & $\begin{array}{l}\text { Aufgabe: fachlich } \\
\text { gute Dienstleistung }\end{array}$ \\
\hline $\begin{array}{l}\text { Privat: } \\
\text { Lebensbewältigung } \\
\text { und Selbstverwirk- } \\
\text { lichung }\end{array}$ & $\begin{array}{l}\text { Interesse: } \\
\text { Kooperation und } \\
\text { Eigenleistungen; } \\
\text { Eigenverantwor- } \\
\text { tung der Bürger, } \\
\text { Überwindung der } \\
\text { Bedarfslage }\end{array}$ & $\begin{array}{l}\text { Interesse: } \\
\text { Zufriedenheit der } \\
\text { Klienten, Bindung, } \\
\text { positives Image }\end{array}$ & $\begin{array}{l}\text { Interesse: Mitarbeit } \\
\text { der Klientel ohne } \\
\text { den Verzicht auf } \\
\text { professionelle Hilfe }\end{array}$ \\
\hline
\end{tabular}

Wer sinnvoll steuern will, muss vier Ebenen in der Sozialen Arbeit deutlich unterscheiden lernen. deren Seite die Kosten dafür möglichst gering zu halten. Dieses liegt zentral im Aufgabenbereich dieser Systemebene: Artikulation einer mehrheitsfähigen Vorstellung von guten Leben, Schaffung von Rahmenbedingungen in Form von Gesetzen, Institutionen und die Alllokation von Ressourcen, also die Bewegung von Geld.

Ein zweites Steuerungsinteresse geht allerdings darüber hinaus und überschreitet ungerechtfertigterweise die Kompetenzgrenze, wobei Kompetenz in beiden Bedeutungen gemeint ist, nämlich als Fähigkeit und Befugnis.

Viele Steuerungsbemühungen, die man an dem Begriff »Gegensteuerung « erkennen kann, gehen von der meistens nicht belegbaren Annahme aus, Organisationen seien ineffizient, Fachleute hätten eine Verschwendungssucht für öffentliche Gelder entwickelt und Bürger würden die Großzügigkeit des Staates ausnutzen. Bis in die Gesetzesformulierungen hinein werden detaillierte operative Anweisungen (zum Beispiel im Bundeskinderschutzgesetz) gegeben, so als ob Professionelle nicht in Lage seien, öffentlich gesetzte Ziele fachlich erreichen zu können.

Öffentlich werden Institutionen und Partner (z. B. »Wohlfahrtsindustrie«, »Wohlfahrtskartell«) diffamiert, egoistischen Zwecken zu dienen und Gelder zu verschwenden. Beispiele dafür können im Schulbereich, im Gesundheitswesen, der Pflege und in der gesamten Sozialen Arbeit gefunden werden. Eine entsprechende Presseerklärung eines Aufsichtsrates eines großen Industriekonzerns, in der dieser seine Mitarbeiter und Zulieferer für den hohen Preis seiner Produkte verantwortlich macht, wäre undenkbar.

Motor der Einflussnahme der oberen Ebene bis auf die Ebene fachlichen Vorgehens ist meistens ein einzelner pressewirksamer Vorfall, zum Beispiel ein spektakulärer Kindstod im Zusammenhang mit einem tatsächlichen oder vermeintlichen Versagens von Helfern. Die Steuerungsstrategie besteht in einer Zunahme an Regelungen und Gesetzen, in dem Infragestellen von Strukturen und in dem Ruf nach mehr Qualität, alles drei aber selten durch ausreichende Ressourcen abgesichert.

Der zweite Gegensteuerungsimpuls wird durch Finanzstatistiken und Haushaltsnöte ausgelöst. Die Steuerungsstra- 
tegien sind hier:

- Budget- oder Entgeltabsenkungen bei gleichbleibenden Ansprüchen

- massive Eingriffe in die Fachlichkeit, so beispielsweise in dem denkwürdigen Beschluss der Oberbürgermeisterin in Halle, alle Heimunterbringungen rückgängig zu machen (HerwigLempp, 2007)

- Verlagerung der Finanzverantwortung auf die operative Ebene, zum Beispiel durch gedeckelte Sozialraumbudgets für kontraktierte Leistungserbringer und

- Rückbau von Rechtsansprüchen.

Intelligentere Lösungen, beispielsweise Doppelarbeiten zu vermeiden, vor Gesetzesvorhaben eine realistischen Folgekostenberechnung vorzunehmen, Prozesskosten für Steuerung und Verwaltung zu senken oder bessere wirkungsorientierte Controlling-Verfahren anzuwenden, ziehen zwar zunehmend in das Repertoire der Institutionen ein, sind aber auf der politisch-administrativen Ebene noch nicht politikleitend.

Dazu ein Beispiel der mittelgroßen Stadt H.: Gute Qualitätsentwicklungen auf der institutionellen Ebene, die sich auch in Wirkungskennzahlen beweisen lässt, einschließlich fallender Kosten bei steigender Wirkung, werden hinweg gewischt durch Behauptungen von Finanzsteuerern, die Ausgaben seien zu hoch im Vergleich mit dem benachbarten Landkreis. Weder die Vergleichbarkeit der Bedarfslagen wird überprüft, noch werden belastbare Qualitätsvergleiche angestellt. Die Abbruchquoten in H. liegen mittlerweile mit 22,5 Prozent deutlich unter den Bundesbenchmark von 29,9 Prozent. Das führt zu einem Rückgang der Fälle, aber auch wegen steigender Durchschnittsverweildauer zu höheren Fallkosten, eine der Lieblingskennzahlen der Finanzsteuerer. Bei den stationären Hilfen ist die Abbruchquote in besagter Stadt 25 Prozent im Vergleich zu durchschnittlich 37,7 Prozent in anderen Jugendämtern. Hilfen, die nicht so oft abbrechen, sind effektiver und führen seltener zu teuren Folgehilfen. Fallzahlen gehen allmählich zurück. Haltbare Hilfen dauern aber auch länger, hier zum Beispiel im Median 19 Monate im Vergleich zu sonst 15 Monaten. Wenn man die Finanzkennzahl durchschnittliche Fall- kosten heranzieht, schneidet $\mathrm{H}$. schlecht ab. Wenn man keine Nutzenkennzahl in die Steuerungslogik einbezieht, kommt man zu völlig unsinnigen Steuerungsentscheidungen.

Eine Untersuchung im Rahmen des von der Kommunalen Gemeinschaftsstelle für Verwaltungsmanagement (KGSt) durchgeführten IKO-Vergleichsrings der wichtigsten deutschen Großstädte, welche Steuerungsstrategien die besten fiskalischen Wirkungen zeitigen, führt zu einem interessanten Ergebnis (Schrapper, 2011): Die höchste Korrelation mit den Kosten- und Fallzahlentwicklungen hat die Organisationsaufmerksamkeit auf fachliche Steuerung. Je besser der fachliche Standard in der Fallsteuerung und der Partizipation der Klienten, desto geringer die Jugendhilfekosten und Leistungsdichten (-0,7 und -0,6). Die höchste Einzelkorrelation besteht zwischen Kosten pro Jungeinwohner und der Durchführung von Evaluationen am Ende einer Hilfe $(-0,78)$. Diese Untersuchung hat den Qualitätsstandard der Verlaufskontrolle noch nicht berücksichtigt. Hätte sie dieses getan, wäre die Korrelation sicherlich noch höher ausgefallen.

\section{"Umstrukturierungen" und "Prozessmanagement"}

Eine der beliebtesten Strategien der Umsteuerung für den Fall, dass Dinge grundsätzlich nicht gut klappen, ist die Umstrukturierung der Institution. Manchmal liegt das eigentliche Problem in überlasteten oder überforderten Mitarbeitenden, ungeeigneten Führungskräften, Konflikten zwischen Abteilungen, unmöglichen Aufträgen mit überzogenen Erwartungen.

Wie auch immer: Am ehesten geht eine Umstrukturierung. Die Idee, dass alles gleich besser werde, weil eine Führungsebene dazukommt oder wegfällt, Dienste eher zentral oder dezentral angesiedelt sind oder eine Abteilung geteilt oder mit einer anderen zusammengelegt werden soll - diese Idee führt nach gemachten Erfahrungen selten zu nachhaltigen Verbesserungen. Im Gegenteil: Ist eine Organisation erst einmal im Wesentlichen mit sich selbst beschäftigt und jagt ein Umsteuerungsversuch den nächsten, schwindet das Vertrauen innerhalb der Organisation und bei Partnern und Kunden.
Eine andere Umsteuerung scheint erfolgreicher zu sein. Es sind die Unternehmensprozesse, die einen Nutzen generieren. Diese daraufhin zu organisieren, dass in klaren Verantwortlichkeiten zielgerichtet und lösungsorientiert gearbeitet wird, ist der Grundgedanke eines recht verstandenen Qualitätsmanagements. Es geht hierbei nicht um eine Standardisierung oder um eine Inflation an Vorschriften und Dokumentation. Prozesse sollten intelligent und effektiv sein. Wohlgeordnete Prozesse machen Fehler weniger wahrscheinlich, beziehungsweise kalkulieren die Früherkennung von Fehlern mit ein. Steuerung wird hier zum Alltagsgeschäft aller Mitarbeitenden

\section{Resümee}

Jedes Bemühen, komplexe lebendige System, also zum Beispiel das Jugendhilfesystem, zu bewegen, zu lenken und $\mathrm{zu}$ steuern, sollte darauf aus sein, effiziente lern- und entwicklungsfähige soziale Gebilde zu schaffen, die in einer Vertrauenskultur für Kunden (im Fall der Jugendhilfe also für junge Menschen und Familien) einen Nutzwert zu bringen, Mitarbeitende als Ressource nutzen und Partner mit Fairness und Respekt zu behandeln.

Wenn es Störungen oder Zielverfehlungen gibt, braucht es weniger einer Gegensteuerung, sondern einer geduldigen, offenen und auch kontroversen Dialogkultur. Dann steuern Systeme schon von allein dagegen. Gilgamesch ist out.

\section{Literatur}

Herwig-Lempp, J. (2007). Der hallesche (Irr-) Weg: Fachkonzept und Dienstanweisung Nr. 93. Forum Jugendhilfe, 13, 311-317.

Schrapper, C. (2011). Wer steuert die Hilfen zur Erziehung? Erfahrungen und Befunde aus 10 Jahren IKO-Netz. Aktuelle Beiträge zur Kinderund Jugendhilfe 80 (S. 29-48). Berlin: Deutsches Institut für Urbanistik.

Sedlacek, T. (2012). Die Ökonomie von Gut und Böse. München: Hanser, Carl.

Tornow, H. (2005). Wirkungsorientierte Steuerung der Sozialen Arbeit. Nachrichtendienst des deutschen Vereins für öffentliche und private Fürsorge, (Heft 6), 1-5. 